\title{
Optimal delivery of anthracycline-based chemotherapy in the adjuvant setting improves outcome of breast cancer patients
}

\author{
Isabel Chirivella $\cdot$ Begoña Bermejo $\cdot$ Amelia Insa $\cdot$ \\ Alejandro Pérez-Fidalgo · Ana Magro · Susana Rosello · \\ Elisa García-Garre · Paloma Martín · Ana Bosch · \\ Ana Lluch
}

Received: 16 January 2008/ Accepted: 9 April 2008/Published online: 8 May 2008

(C) Springer Science+Business Media, LLC. 2008

\begin{abstract}
To evaluate the dose-response effect of an adjuvant anthracycline-based non-taxane chemotherapy in early breast cancer patients. This was a retrospective database analysis. Selection criteria included patients treated for early breast cancer from years 1980 to 2000 with an adjuvant anthracycline-based non-taxane chemotherapy. The delivery of chemotherapy was assessed through the number of delayed cycles, the number of delayed days and the relative dose intensity (RDI) administered $(\geq 85 \%,<85 \%)$. Seven hundred and ninety-three breast cancer patients were included. The Kaplan-Meier disease-free survival (DFS) was affected by the number of delayed cycles $(P<0.0001)$, the number of delayed days $(P<0.0001)$ and the RDI $(P=0.0029)$. The KaplanMeier overall survival (OS) was also affected by the number of delayed cycles $(P=0.0008)$ and days $(P=0.0115)$, as well as the RDI $(P=0.0055)$. The Cox regression models showed that, when the number of nodes affected and the hormonal receptor status were controlled, all the study variables maintained their significance on DFS, but not on OS. The dose-response effect is a crucial factor in the administration of anthracycline-based nontaxane schedules for the adjuvant treatment of early breast cancer. Delays and/or reductions of chemotherapy should be avoided if possible to achieve the maximal benefit.
\end{abstract}

Keywords Dose-response effect - Anthracycline-based . Non-taxane $\cdot$ Adjuvant chemotherapy

I. Chirivella - B. Bermejo - A. Insa - A. Pérez-Fidalgo ·

A. Magro - S. Rosello · E. García-Garre · P. Martín .

A. Bosch · A. Lluch $(\square)$

Medical Oncology Service, Hospital Clínico Universitario,

Avda. Blasco Ibáñez, 17, 46011 Valencia, Spain

e-mail: ana.lluch@uv.es

\section{Introduction}

In the 1970s, it was first reported that patients with early breast cancer who received cyclophosphamide, methotrexate and fluorouracil (CMF) at full doses in the adjuvant setting had an improved disease-free survival (DFS) and overall survival (OS) compared with those patients who only underwent surgery [1]. Three decades later, the benefits obtained by this patient group were still evident [2]. Later, the Spanish Breast Cancer Research Group (GEICAM) demonstrated that six cycles of fluorouracil, doxorubicin and cyclophosphamide (FAC) were superior to six cycles of CMF in the adjuvant treatment of operable breast cancer [3]. These findings were confirmed by the meta-analysis of the Early Breast Trialist's Collaborative Group (EBTCG), in which anthracycline-based combinations reduced the mortality of breast cancer patients, irrespective of the estrogen receptor and/or nodal status, in comparison with CMF schedules [4].

In spite of the strong evidence that supports the use of adjuvant chemotherapy, conflicting results have been reported about the relationship between chemotherapy dose and treatment response [5]. Bonnadona et al. were the first to report a clear dose-response effect during the administration of CMF, particularly in the adjuvant setting [6]. From that point, several retrospective analyses found similar improvements in outcomes [7,8], whereas others reported no dose-response effect $[9,10]$. However, the dose groups used in these analyses frequently varied from those used originally by Bonadonna. Using the same dose level groupings ( $\geq 85 \%, 65-84 \%$, and $<65 \%$ ) as the first study, a later study again showed that the dose level of CMF administered was an important prognosis factor in node-positive breast cancer patients. It was concluded that patients who received less than $65 \%$ of the prescribed CMF dose had a significantly worse outcome [11]. 
Based on these findings, we considered it to be of interest to evaluate whether this dose-response effect reported for CMF administration was also true with anthracycline-based non-taxane schedules in the adjuvant treatment of early breast cancer patients. We retrospectively analysed the impact of chemotherapy delivery on DFS and OS at 10 years. The variables selected to assess chemotherapy delivery included not only the dose level administered, but also administration delays.

\section{Methods}

\section{Study design}

This was a retrospective database analysis performed in June 2007. Database was created in 1980. Since then, clinical data for all patients treated at the Hospital Clinico Universitario of Valencia (Spain) for breast cancer have been entered into this database.

Confidentiality of patients' data was maintained throughout the study. Data extraction was performed by two data managers. Four independent medical oncologists verified $15 \%$ of the extracted data against the original database to confirm the accuracy of the data extraction.

\section{Study procedures}

Inclusion criteria to be included in this retrospective analysis were to have a diagnosis of early breast cancer (stages I-IIIA) from January 1980 through December 2000, the primary treatment of the disease being a surgical procedure and an anthracycline-based non-taxane chemotherapy in the adjuvant setting. For each selected patient, the database included information about the schedule of chemotherapy given together with drug dosage and the quantitative and qualitative description of treatment delays and/or reductions performed. Patients who had to interrupt chemotherapy due to toxicity or any other reason were excluded from this analysis. To avoid poor quality data, patients not diagnosed in this hospital were also excluded.

In the adjuvant setting, patients could have received any of the following schedules: 3 cycles of doxorubicin $\left(\mathrm{A}, 30 \mathrm{mg} / \mathrm{m}^{2}\right.$ ) plus cyclophosphamide $\left(\mathrm{C}, 600 \mathrm{mg} / \mathrm{m}^{2}\right.$ ) every 21 days if they were staged with a T1NOM0 disease $(3 \times \mathrm{AC}) ; 8$ cycles of the same schedule if they had a T2NOM0 disease $(8 \times \mathrm{AC})$; or 3 cycles of $\mathrm{A}\left(50 \mathrm{mg} / \mathrm{m}^{2}\right)$, $\mathrm{C}\left(600 \mathrm{mg} / \mathrm{m}^{2}\right), 5$-fluorouracil $\left(\mathrm{F}, 600 \mathrm{mg} / \mathrm{m}^{2}\right)$ and methotrexate $\left(\mathrm{M}, 30 \mathrm{mg} / \mathrm{m}^{2}\right)$ every 21 days followed by 5 cycles of FAC $\left(600 / 40 / 600 \mathrm{mg} / \mathrm{m}^{2}\right)$ every 21 days $(3 \times$ FAC-M $\rightarrow 5 \times$ FAC) for those patients who had T3N0 and T1-1N1-2 disease. Tamoxifen was started after chemotherapy completion and continued for 5 years in all patients with hormone receptor-positive tumors. Radiotherapy was initiated within 4 weeks after the last cycle of chemotherapy in all patients who had undergone breastconserving surgery or had got a tumor size $>5 \mathrm{~cm}$ or $\geq 4$ lymph nodes affected

Standard dose modification criteria were in place at the hospital. If the neutrophil count fell to $<1.5 \times 10^{9} / 1$ or platelets $<100 \times 10^{9} / 1$, chemotherapy was delayed 5-7 days, but reintroduced upon recovery (neutrophil count $>1.5 \times$ $10^{9} / 1$ or platelets $>100 \times 10^{9} / \mathrm{l}$ ) without any dosage modification. Granulocyte colony-stimulating factor (G-CSF) support was not administered to any of these patients. Chemotherapy was also delayed in the case of any grade 3/4 non-hematologic toxicity, and restarted when it improved to grade 1 . If toxicity persisted, dosages were reduced to $75 \%$. If toxicity still persisted after three weeks, chemotherapy was definitely interrupted.

Data extracted included patient age, year of diagnosis, tumor stage, histologic grade, as well as menopausal and hormonal receptor status. Other treatment-related data were extracted such as the mean percentage of administered dose throughout the cycles, the number of delayed chemotherapy cycles, the number of days of delay in chemotherapy administration and the last day of follow-up and any event (disease recurrence or death) that occurred to the patient during the follow-up period.

\section{Statistical analysis}

The primary objective of this analysis was to evaluate whether the optimal delivery of an anthracycline-based chemotherapy in the adjuvant setting could impact on DFS and OS at 10 years. OS at 10 years was defined as being alive 10 years after cancer diagnosis. Similarly, DFS at 10 years was defined as being alive, with no disease recurrence, 10 years after cancer diagnosis.

Three variables were chosen to assess the delivery of chemotherapy to the patient: the number of delayed cycles, the number of delayed days, and the RDI administered. The number of delayed cycles was based on whether the patient had more than 2 cycles with $\geq 3$ days of delay with respect to the planned schedule $(\leq 2$ delayed cycles, $>2$ delayed cycles). The number of delayed days during treatment administration was based on whether the patient's chemotherapy had to be delayed more than 14 days overall or not $(<15$ delayed days, $\geq 15$ delayed days). Finally, the RDI was based on whether or not the patient's RDI was less than $85 \%(\geq 85 \%,<85 \%)$. RDI was calculated as the mean percentage of administered dose throughout the entire treatment multiplied by the ratio of the number of treatment days as planned to the number of treatment days as planned plus the number of delayed days. 
Descriptive statistical data evaluation was based on medians and standard deviations (SD) for continuous endpoints, and on absolute and relative frequencies with 95\% confidence intervals (CI) for categorical variables. Dichotomous categorical variables were compared using a 2 -sided log rank test. The survival analysis consisted of an overall analysis of DFS and OS and a Kaplan-Meier estimation for each outcome according to the three study variables. Stratification of the Kaplan-Meier estimation by previously defined clinically relevant covariables such as the number of affected lymph nodes $(0,1-3,4-10,>10)$ and the hormonal status (estrogen and progesterone receptors) was also performed. Finally, a Cox regression analysis to investigate the effect of study variables on DFS and OS while controlling previously defined covariables was performed. Statistical significance was set at $P<0.05$.

\section{Results}

Of the 1,790 breast cancer patients included in the initial database, 793 were included in this analysis. The reasons to exclude 997 patients were the following: $263(26 \%)$ patients because of the lack of important clinical data, 232 (23\%) were participating in different clinical trials with taxanes or high-dose chemotherapy, $229(23 \%)$ were treated with hormonal therapy only, $178(18 \%)$ received neoadjuvant chemotherapy and $95(10 \%)$ were treated with CMF in the adjuvant setting. Patient characteristics at baseline are shown in Table 1. The majority of patients were diagnosed and treated from 1985 to 1989 (36\%) and from 1990 to 1994 (31\%), and had stage II disease (50\%) with a histologic grade II (74\%). Forty-seven percent of patients did not have lymph node involvement, whereas $32 \%$ had $1-3,17 \%$ had $4-10$, and $4 \%$ of patients had more than 10 nodes affected. An almost equal percentage of patients were pre- or perimenopausal $(48 \%)$ and postmenopausal $(52 \%)$.

Impact of suboptimal chemotherapy delivery on DFS and OS

With a median follow-up period of almost 10 years for the entire study population, the median DFS was 8.30 years (95\% CI: 7.95-9.10) and the median OS was 9.42 years (95\% CI: 8.96-10.11). At 10 years, the probability of surviving without recurrence of the disease was $66 \%$ (95\% CI: 63-70) and the probability of being alive, with or without disease recurrence, was $77 \%$ (95\% CI: 73-80).

As shown in Table 2, the Kaplan-Meier DFS was significantly affected by the number of delayed cycles (HR: 2.07, 95\% CI: $1.61-2.67, P<0.0001)$, the number of delayed days (HR: $1.77,95 \% \mathrm{CI}: 1.38-2.28, P<0.0001$ )
Table 1 Patient characteristics at diagnosis

\begin{tabular}{|c|c|c|}
\hline Characteristics & $N$ & $\%$ \\
\hline \multicolumn{3}{|l|}{ Age, years $(n=793)$} \\
\hline Median (range) & $51(21-79)$ & \\
\hline \multicolumn{3}{|l|}{ Treatment period $(n=789)$} \\
\hline 1980-1984 & 86 & 11 \\
\hline $1985-1989$ & 285 & 36 \\
\hline 1990-1994 & 246 & 31 \\
\hline 1995-2000 & 172 & 22 \\
\hline \multicolumn{3}{|l|}{ Tumor stage $(n=782)$} \\
\hline $\mathrm{I}$ & 182 & 23 \\
\hline II & 388 & 50 \\
\hline IIIA & 212 & 27 \\
\hline \multicolumn{3}{|l|}{ Histological grade $(n=684)$} \\
\hline I & 65 & 9 \\
\hline II & 506 & 74 \\
\hline III & 113 & 17 \\
\hline \multicolumn{3}{|l|}{ Lymph nodes involvement $(n=785)$} \\
\hline 0 & 368 & 47 \\
\hline $1-3$ & 248 & 32 \\
\hline $4-10$ & 135 & 17 \\
\hline$>10$ & 34 & 4 \\
\hline \multicolumn{3}{|l|}{ Hormonal receptor status } \\
\hline $\mathrm{ER}+(n=594)$ & 332 & 56 \\
\hline $\mathrm{PR}+(n=593)$ & 305 & 51 \\
\hline \multicolumn{3}{|l|}{ Menopausal status $(n=754)$} \\
\hline Pre-/Perimenopausal & 360 & 48 \\
\hline Postmenopausal & 394 & 52 \\
\hline \multicolumn{3}{|l|}{ Adjuvant chemotherapy $(n=793)$} \\
\hline 3 cycles of $\mathrm{AC}$ & 153 & 19 \\
\hline 8 cycles of $\mathrm{AC}$ & 202 & 26 \\
\hline 3 cycles of FAC-M $>5$ cycles of FAC & 438 & 55 \\
\hline \multicolumn{3}{|l|}{ Chemotherapy administration } \\
\hline \multicolumn{3}{|l|}{ Delayed cycles $(n=793)$} \\
\hline$\leq 2$ delayed cycles & 581 & 73 \\
\hline$>2$ delayed cycles & 212 & 27 \\
\hline \multicolumn{3}{|l|}{ Delayed days $(n=793)$} \\
\hline$<15$ days & 560 & 71 \\
\hline$\geq 15$ days & 233 & 29 \\
\hline \multicolumn{3}{|l|}{ RDI $(n=791)$} \\
\hline$\geq 85 \%$ & 698 & 88 \\
\hline$<85 \%$ & 93 & 12 \\
\hline
\end{tabular}

ER: estrogen receptor; PR: progesterone receptor; A: doxorubicin; C: cyclophosphamide; F: 5-fluorouracil; M: methotrexate; RDI: relative dose intensity

and the RDI (HR: $1.65,95 \%$ CI: $1.18-2.30, P=0.0029$ ). Thus, patients with delays in scheduled chemotherapy (counted as $\geq 15$ delayed days or as $>2$ delayed cycles) as well as patients who received a reduced RDI $(<85 \%)$ had significantly lower probability of survival without 
Table 2 Impact of chemotherapy delivery on disease-free survival and overall survival
*Adjusted by affected lymph nodes, stage and progesterone receptors

\begin{tabular}{|c|c|c|c|c|c|c|}
\hline \multirow[t]{2}{*}{ Characteristics } & \multirow{2}{*}{$\begin{array}{l}\text { Patients } \\
(\%)\end{array}$} & \multirow{2}{*}{$\begin{array}{l}\text { Events } \\
(\%)\end{array}$} & \multirow{2}{*}{$\begin{array}{l}\text { Censored } \\
(\%)\end{array}$} & \multirow{2}{*}{$\begin{array}{l}\text { Log Rank } \\
(P)\end{array}$} & \multicolumn{2}{|c|}{ HR (CI 95\%) } \\
\hline & & & & & Unadjusted & Adjusted* \\
\hline \multicolumn{7}{|c|}{ Disease free survival } \\
\hline \multicolumn{7}{|c|}{ Delayed cycles $(n=792)$} \\
\hline $\begin{array}{l}\leq 2 \text { delayed } \\
\text { cycles }\end{array}$ & 580 & $145(25)$ & $435(75)$ & 32.97 & 2.07 & 1.64 \\
\hline $\begin{array}{l}>2 \text { delayed } \\
\text { cycles }\end{array}$ & 212 & $101(48)$ & $111(52)$ & $(P<0.0001)$ & $(1.61-2.67)$ & $(1.21-2.22)$ \\
\hline \multicolumn{7}{|c|}{ Delayed days $(n=792)$} \\
\hline$<15$ days & 559 & $144(26)$ & $415(74)$ & 20.05 & 1.77 & 1.41 \\
\hline$\geq 15$ days & 233 & $102(44)$ & $131(56)$ & $(P<0.0001)$ & $(1.38-2.28)$ & $(1.04-1.90)$ \\
\hline \multicolumn{7}{|c|}{ RDI administered $(n=790)$} \\
\hline$\geq 85 \%$ & 697 & $203(29)$ & $494(71)$ & 8.86 & 1.65 & 1.57 \\
\hline$<85 \%$ & 93 & $42(45)$ & $51(55)$ & $(P=0.0029)$ & $(1.18-2.30)$ & $(1.06-2.31)$ \\
\hline \multicolumn{7}{|l|}{ Overall survival } \\
\hline \multicolumn{7}{|c|}{ Delayed cycles $(n=793)$} \\
\hline $\begin{array}{l}\leq 2 \text { delayed } \\
\text { cycles }\end{array}$ & 581 & $102(18)$ & $479(82)$ & 11.34 & 1.70 & 1.21 \\
\hline $\begin{array}{l}>2 \text { delayed } \\
\text { cycles }\end{array}$ & 212 & $64(30)$ & $148(70)$ & $(P=0.0008)$ & $(1.24-2.33)$ & $(0.83-1.76)$ \\
\hline \multicolumn{7}{|c|}{ Delayed days $(n=793)$} \\
\hline$<15$ days & 560 & $101(18)$ & $459(82)$ & 6.39 & 1.49 & 1.02 \\
\hline$\geq 15$ days & 233 & $65(28)$ & $168(72)$ & $(P=0.0115)$ & $(1.09-2.04)$ & $(0.70-1.48)$ \\
\hline \multicolumn{7}{|c|}{ RDI administered $(n=791)$} \\
\hline$\geq 85 \%$ & 698 & $135(19)$ & $563(81)$ & 7.71 & 1.73 & 1.45 \\
\hline$<85 \%$ & 93 & $31(33)$ & $62(67)$ & $(P=0.0055)$ & $(1.17-2.55)$ & $(0.90-2.32)$ \\
\hline
\end{tabular}

recurrence of the disease at 10 years (Fig. 1). The KaplanMeier DFS analysis was statistically significant when data was stratified by the number of lymph nodes affected and the hormonal receptor status (estrogen or progesterone receptors), with $P$ values in all cases $<0.05$.

The Kaplan-Meier OS was also significantly affected by the number of delayed cycles (HR: $1.70,95 \%$ CI: 1.24-2.33, $P=0.0008$ ), the number of delayed days (HR: $1.49,95 \%$
CI: $1.09-2.04, P=0.0115)$ and the RDI (HR: $1.73,95 \%$ CI: $1.17-2.55, P=0.0055)$. Patients who experienced treatment delays (counted as $\geq 15$ delayed days or $>2$ delayed cycles) as well as patients who received a reduced RDI ( $<85 \%$ ) had a significantly lower probability of being alive, with or without recurrence of the disease, at 10 years (Fig. 2). When the data obtained from the Kaplan-Meier OS analysis were stratified by the number
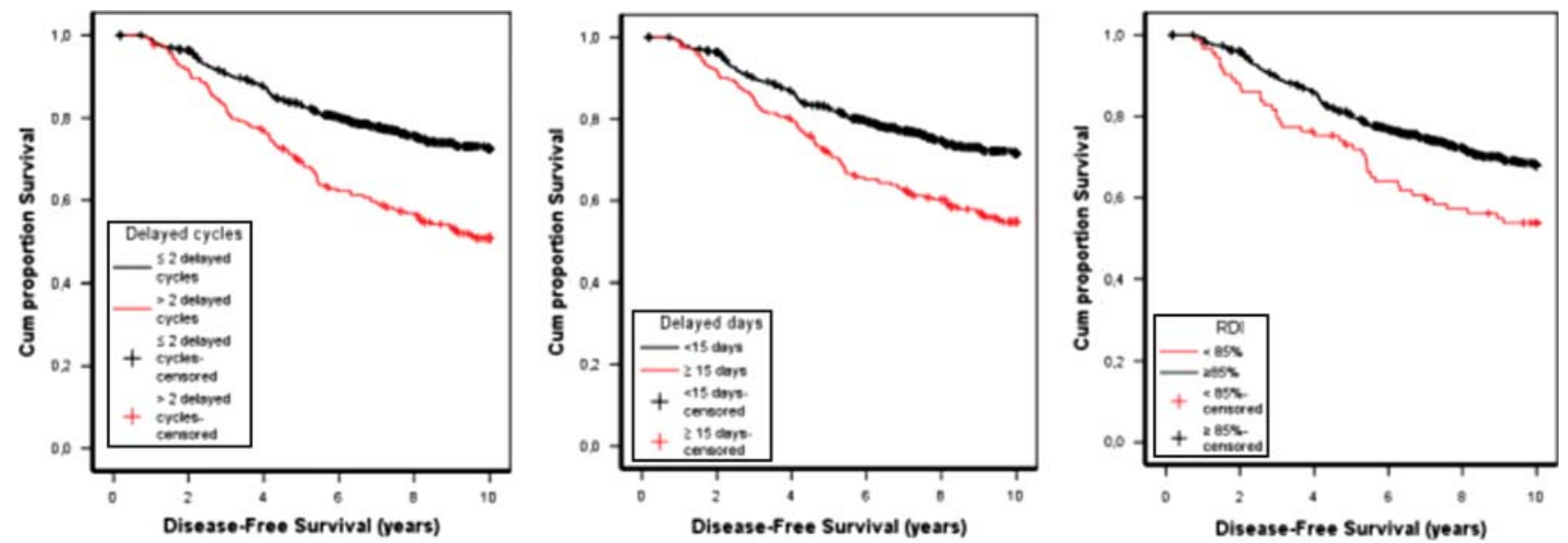

Fig. 1 Impact of study variables on disease-free survival: (a) Number of delayed cycles ( $\leq 2$ cycles, $>2$ cycles); (b) Number of delayed days ( $<15$ days, $\geq 15$ days); (c) RDI ( $\geq 85 \%,<85 \%)$ 

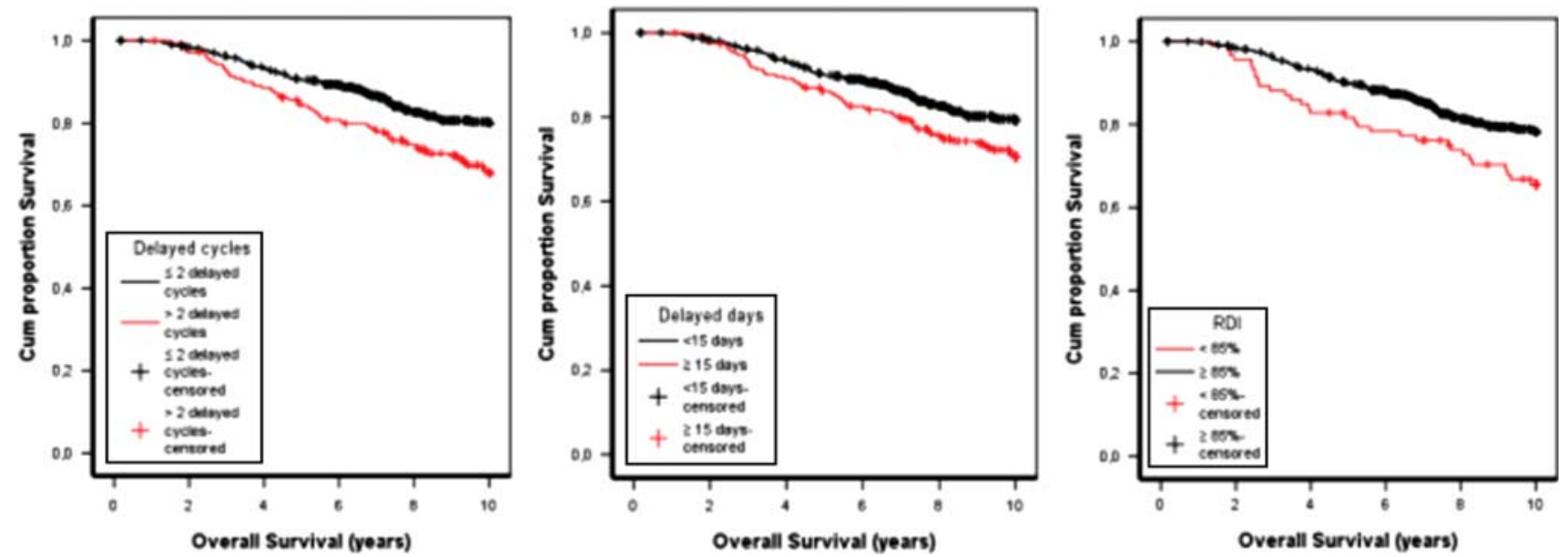

Fig. 2 Impact of study variables on overall survival: (a) Number of delayed cycles ( $\leq 2$ cycles, $>2$ cycles); (b) Number of delayed days ( $<15$ days, $\geq 15$ days); (c) RDI ( $\geq 85 \%,<85 \%)$

of nodes affected and the hormonal receptor status, the only study variable that maintained its significance with any of the covariables evaluated was the number of delayed cycles $(\leq 2$ cycles, $>2$ cycles). The number of delayed days ( $<15$ days, $\geq 15$ days) did not keep significance with any of the clinically relevant covariables. Lastly, RDI ( $\geq 85 \%,<85 \%)$ had a statistically significant impact on OS when stratification was performed by the number of lymph nodes affected (HR: 1.61, 95\% CI: $1.09-2.39, P=0.0128)$, but not when stratification was performed by estrogen receptors (HR: $1.57,95 \% \mathrm{CI}$ : $0.98-2.51, P=0.0559)$ or progesterone receptors (HR: 1.56, 95\% CI: $0.98-2.49, P=0.0592$ ), although in both cases there was a clear trend.

\section{Cox regression models for DFS and OS}

As shown in Table 2, the Cox regression models showed that, when other clinically relevant disease characteristics such as number of nodes affected and hormonal receptor status were controlled, the three main study variables maintained significance on DFS (for $>2$ cycles, HR: 1.64, 95\% CI: $1.21-2.22, P=0.001$ ), (for $\geq 15$ days, HR: 1.41 , 95\% CI: 1.04-1.90, $P=0.027$ ), (for RDI $<85 \%$, HR: 1.57, 95\% CI: $1.06-2.31, P=0.023$ ).

However, when modeling OS by Cox regression analysis controlling for the clinically relevant disease characteristics, the three study variables did not show statistical significance.

\section{Discussion}

We have retrospectively evaluated whether the optimal delivery of an anthracycline-based non-taxane chemotherapy could improve DFS and OS in the adjuvant setting of early breast cancer patients. Our results showed that those patients with delays in scheduled chemotherapy or who received a reduced RDI had significantly lower probability of being alive, with or without recurrence of the disease, at 10 years.

These results are similar to those observed by two previous retrospective analyses performed with $\mathrm{CMF}$ in the adjuvant setting of breast cancer $[6,11]$. In the first study [6], CMF was found to be effective when given in a full, or nearly full, dose ( $\geq 85 \%$ of the planned dose). Within each dose level, the results were influenced by the number of axillary nodes involved but not by the menopausal status. In another study [11], a better OS and DFS were observed in the intermediate dose level group (65-84\%) in comparison with the lower $(<65 \%)$ dose level group. These results were consistent within different patient subgroups (defined by menopausal status and estrogen receptor status). In the case of nodal status, differences remained only in the case of patients with 1-3 positive nodes.

In the present analysis, DFS remained statistically different for the three study variables within all patient subgroups (defined by the number of nodes affected and the hormonal receptor status). However, only the number of delayed cycles had a significant impact on OS within all patients subgroups.

Our results also support the idea that the modest results obtained with many cytotoxic agents in the adjuvant setting may be attributed, in part, to the sub-optimal administration of chemotherapy in which thresholds of dose intensity are not reached [12-14]. In the specific case of obese patients, this seems to be a common practice that indeed has a detrimental effect on their outcome [15-18]. The practice of prolonged dose reductions or delays to restrict toxicity should be avoided [19]. 
In spite of the important results reported from this retrospective analysis, it is possible that there may be some bias [20]. In particular, patients who did not have to delay chemotherapy or to reduce RDI may have different clinical characteristics than those patients who did not. However, the Cox regression models performed during this study showed that, when the number of lymph nodes affected and the hormonal receptor status were controlled, the three study variables maintained their significance on DFS, although not for OS. Indeed, within the clinical characteristics that may affect our results, the number of lymph nodes remains the strongest predictive factor of breast cancer recurrence and survival $[8,10]$.

In conclusion, the results of this retrospective analysis indicate that the dose-response effect is a crucial factor in the administration of anthracycline-based non-taxane schedules for the adjuvant treatment of early breast cancer. Thus, delays and/or reductions of chemotherapy should be avoided whenever possible to achieve the maximal benefit.

Acknowledgements The authors acknowledge the work of Salutis (Barcelona, Spain) in carrying out the statistical analysis of the study, Dr. Beatriz Gil-Alberdi from HealthCo (Madrid, Spain) for assistance in the preparation of the manuscript, and Amgen SA Spain for financial support.

\section{References}

1. Bonadonna G, Brusamolino E, Valagussa P et al (1976) Combination chemotherapy as an adjuvant treatment in operable breast cancer. N Engl J Med 294:405-410

2. Bonadonna G, Moliterni A, Zambetti M et al (2005) 30 years' follow up of randomised studies of adjuvant CMF in operable breast cancer: cohort study. BMJ 330:217

3. Martin M, Villar A, Sole-Calvo A et al (2003) Doxorubicin in combination with fluorouracil and cyclophosphamide (i.v. FAC regimen, day 1,21 ) versus methotrexate in combination with fluorouracil and cyclophosphamide (i.v. CMF regimen, day 1,21) as adjuvant chemotherapy for operable breast cancer: a study by the GEICAM group. Ann Oncol 14:833-842

4. Early Breast Cancer Trialists' Collaborative Group (EBCTCG) (2005) Effects of chemotherapy and hormonal therapy for early breast cancer on recurrence and 15-year survival: an overview of the randomised trials. Lancet 365:1687-1717

5. Henderson IC, Hayes DF, Gelman R (1988) Dose-response in the treatment of breast cancer: a critical review. J Clin Oncol 6:15011515
6. Bonadonna G, Valagussa P (1981) Dose-response effect of adjuvant chemotherapy in breast cancer. N Engl J Med 304: $10-15$

7. Senn HJ, Barett-Mahler AR, Jungi WF et al (1989) Adjuvant chemoimmunotherapy with $\mathrm{LMF}+\mathrm{BCG}$ in node-negative and node-positive breast cancer patients: 10 year results. Eur J Cancer Clin Oncol 25:513-525

8. Velez-Garcia E, Carpenter JT Jr, Moore M et al (1992) Postsurgical adjuvant chemotherapy with or without radiotherapy in women with breast cancer and positive axillary nodes: a SouthEastern Cancer Study Group (SEG) Trial. Eur J Cancer 28A: $1833-1837$

9. Howell A, Rubens RD, Bush H et al (1984) A controlled trial of adjuvant chemotherapy with melphalan versus cyclophosphamide, methotrexate, and fluorouracil for breast cancer. Recent Results Cancer Res 96:74-89

10. Ahmann DL, O'Fallon JR, Scanlon PW et al (1982) A preliminary assessment of factors associated with recurrent disease in a surgical adjuvant clinical trial for patients with breast cancer with special emphasis on the aggressiveness of therapy. Am J Clin Oncol 5:371-381

11. Colleoni M, Price K, Castiglione-Gertsch M et al (1998) Doseresponse effect of adjuvant cyclophosphamide, methotrexate, 5-fluorouracil (CMF) in node-positive breast cancer. International Breast Cancer Study Group. Eur J Cancer 34:1693-1700

12. Gurney $\mathrm{H}$ (2002) How to calculate the dose of chemotherapy. $\mathrm{Br}$ J Cancer 86:1297-1302

13. Lyman GH, Dale DC, Crawford J (2003) Incidence and predictors of low dose-intensity in adjuvant breast cancer chemotherapy: a nationwide study of community practices. J Clin Oncol 21:45244531

14. Budman DR (2004) Dose and schedule as determinants of outcomes in chemotherapy for breast cancer. Semin Oncol 31:3-9

15. Poikonen P, Blomqvist C, Joensuu H (2001) Effect of obesity on the leukocyte nadir in women treated with adjuvant cyclophosphamide, methotrexate, and fluorouracil dosed according to body surface area. Acta Oncol 40:67-71

16. Rosner GL, Hargis JB, Hollis DR et al (1996) Relationship between toxicity and obesity in women receiving adjuvant chemotherapy for breast cancer: results from cancer and leukemia group B study 8541. J Clin Oncol 14:3000-3008

17. Colleoni M, Li S, Gelber RD et al (2005) Relation between chemotherapy dose, oestrogen receptor expression, and bodymass index. Lancet 366:1108-1110

18. Goodwin PJ, Boyd NF (1990) Body size and breast cancer prognosis: a critical review of the evidence. Breast Cancer Res Treat 16:205-214

19. Wood WC, Budman DR, Korzun AH et al (1994) Dose and dose intensity of adjuvant chemotherapy for stage II, node-positive breast carcinoma. N Engl J Med 330:1253-1259

20. Redmond C, Fisher B, Wieand HS (1983) The methodologic dilemma in retrospectively correlating the amount of chemotherapy received in adjuvant therapy protocols with disease-free survival. Cancer Treat Rep 67:519-526 